\title{
Plasticity
}

\section{Intrauterine Growth Restriction: Effects on Neural Precursor Cell Proliferation and Angiogenesis in the Foetal Subventricular Zone}

\author{
Mary Tolcos ${ }^{\mathrm{a}-c}$ Rachel Markwick ${ }^{\mathrm{a}}$ Rachael O'Dowd ${ }^{\mathrm{a}}$ Veronica Martin ${ }^{\mathrm{a}}$ \\ Ann Turnley ${ }^{a}$ Sandra Rees ${ }^{\text {a }}$ \\ ${ }^{a}$ Department of Anatomy and Neuroscience, University of Melbourne, Melbourne, Vic., ${ }^{\text {b}}$ The Ritchie Centre, \\ MIMR-PHI Institute of Medical Research and Monash University, and 'Department of Obstetrics and Gynaecology, \\ Monash University, Clayton, Vic., Australia
}

\section{Key Words}

Chronic placental insufficiency - Brain development .

Hypoxia · Neurogenesis · Cerebral cortex · Gliogenesis .

Microvasculature $\cdot$ Neurogenic niche

\begin{abstract}
Exposure to adverse prenatal factors can result in abnormal brain development, contributing to the aetiology of several neurological disorders. Intrauterine insults could occur during neurogenesis and gliogenesis, disrupting these events. Here we investigate the effects of chronic placental insufficiency (CPI) on cell proliferation and the microenvironment in the subventricular zone (SVZ). At 30 days of gestation (DG; term $67 \mathrm{DG}), \mathrm{CPI}$ was induced in pregnant guinea pigs via unilateral uterine artery ligation to produce growth-restricted (GR) foetuses $(n=7)$; controls $(n=6)$ were from the unoperated horn. At $60 \mathrm{DG}$, foetal brains were stained immunohistochemically to identify proliferating cells (Ki67), immature neurons (polysialylated neuronal cell adhesion molecule), astrocytes (glial fibrillary acidic protein), microglia (ionised calcium-binding adaptor molecule-1, lba-1) and the microvasculature (von Willebrand factor) in the SVZ. There was no overall difference $(p>0.05)$ in the total number of Ki67-immunoreactive (IR) cells, the percentage of SVZ occupied by blood vessels or the density of Iba-1-IR microglia
\end{abstract}

in control versus GR foetuses. However, regression analysis across both groups revealed that both the number of Ki67-IR cells and the percentage of SVZ occupied by blood vessels in the ventral SVZ were negatively correlated $(p<0.05)$ with brain weight. Furthermore, in the SVZ (dorsal and ventral) the density of blood vessels positively correlated $(p<0.05)$ with the number of Ki67-IR cells. Double-labelling immunofluorescence suggested that the majority of proliferating cells were likely to be neural precursor cells. Thus, we have demonstrated an association between angiogenesis and neurogenesis in the foetal neurogenic niche and have identified a window of opportunity for the administration of trophic support to enhance a neuroregenerative response.

๑) 2015 S. Karger AG, Basel

\section{Introduction}

Intrauterine growth restriction (IUGR) can have immediate and life-long consequences to the intellectual [1], cognitive [1] and motor function [2,3] of an individual. IUGR can occur for a number of reasons but is most commonly due to placental insufficiency which causes dysfunction to the foetal-placental unit, leading to chronic foetal hypoxaemia, slowing of foetal growth and compromise to several organ systems, including the developing

\section{KARGER 125}

(c) 2015 S. Karger AG, Base

0378-5866/15/0375-0453\$39.50/0

E-Mail karger@karger.com

www.karger.com/dne
Dr. Mary Tolcos

The Ritchie Centre

MIMR-PHI Institute of Medical Research and Monash University

27-31 Wright Street, Clayton, VIC 3168 (Australia)

E-Mail mary.tolcos@ mimr-phi.org 
brain $[4,5]$. Although the brain is spared relative to other organs as a result of adaptive mechanisms such as the redistribution of blood flow [6], key neurodevelopmental and cellular processes are likely to be affected, and these may underlie the adverse neurological outcome. There is a need to identify cellular targets that could be exploited therapeutically to improve the outcome for these individuals. As IUGR occurs during the period of neurogenesis and the initial phase of neural process growth and connectivity, these aspects of development are likely to be affected. Indeed, in a guinea pig model of IUGR induced by chronic placental insufficiency (CPI) throughout the second half of pregnancy, we have already shown that, postnatally, specific populations of neurons are reduced in number [7], neural process growth is decreased [7] and neocortical volume is reduced [8]. Thus CPI clearly has adverse effects on neural development which are evident after birth, but we do not know whether the brain also attempts to mount a regenerative response prenatally which could be targeted to ameliorate neurodevelopment. A possible site of such a response is the subventricular zone (SVZ) - the neurogenic zone in the forebrain from which neurons and neuroglia are generated in later stages of foetal development [9]. This area has been shown to respond to hypoxic insults, albeit in the neonate, with increased cell proliferation [10-14], although there have also been reports of decreased proliferation [15-17]. Specifically, the aims of this study were as follows: (1) to determine the ongoing effects of CPI on cell proliferation in the foetal SVZ; (2) to characterise the phenotype of the proliferating cells; (3) to identify alterations to specific cellular components (astrocytes and microglia) of the compromised microenvironment, and (4) to identify any association between the cerebral microvasculature and cell proliferation in the compromised SVZ.

\section{Materials and Methods}

Experiments were approved by the University of Melbourne Animal Experimentation and Ethics Committee and were carried out in accordance with the National Health and Medical Research Council of Australia and international guidelines.

\section{Surgery}

CPI was induced by unilateral uterine artery ligation in datemated Dunkin-Hartley guinea pigs $(\mathrm{n}=9)$, as described previously $[8,18]$. At $28-30$ days of gestation (DG; term $\sim 67$ days) pregnant guinea pigs were anaesthetised (ketamine, $40 \mathrm{mg} / \mathrm{kg}$, i.m.; Ilium Laboratories, Vic., Australia and Xylazil, $6 \mathrm{mg} / \mathrm{kg}$, i.m.; Troy Laboratories, N.S.W., Australia), and a midline incision was made in the abdominal wall exposing the peritoneal cav- ity. The mesometrium of one uterine horn was located and the uterine artery was ligated near the cervical end of the arterial arcade; the ligature remained in place for the duration of the pregnancy. After suturing, the incision was treated with a Chlorhex C/ethanol solution and a topical antibiotic powder (Cicatrin ${ }^{\circledR}$ powder; GlaxoSmithKline, Middlesex, UK). Foetal reabsorptions occurred in $11 \%$ ( 1 out of 9 ) of dams following uterine artery ligation; this did not impact on the growth of the foetus within the ligated horn. Foetuses from the unligated horn were used as controls.

Animals

Foetuses were delivered by caesarean section, under deep anaesthesia (Lethabarb; sodium pentobarbitone, $130 \mathrm{mg} / \mathrm{kg}$, i.p; Virbac Pty. Ltd., Sydney, N.S.W., Australia) between 60 and 62 DG. Foetuses and placentae were weighed and the crown-rump length measured. Foetal guinea pigs were considered to be GR if their mean body weight and crown-rump length were 2 standard deviations (SD) below the mean weight for age-matched controls and their mean brain-to-body weight ratio was 2 SD above the mean ratio for age-matched controls [19].

\section{Perfusion and Tissue Preparation}

Foetal guinea pigs (60-62 DG; control: $n=6$; GR: $n=7$ ) were transcardially perfused with saline and $4 \%$ PFA (paraformaldehyde) in $0.1 \mathrm{M} \mathrm{PB}$ (phosphate buffer; $\mathrm{pH} 7.4$ ). Brain weights were recorded, and the tissue was post-fixed in $4 \%$ PFA for $4 \mathrm{~h}$ at $4^{\circ} \mathrm{C}$. The left cerebral hemisphere was embedded in paraffin and serially sectioned $(8 \mu \mathrm{m})$ from the anterior pole of the lateral ventricle (LV) to the commencement of the hippocampus. Every 10th section was then collected for immunohistochemistry; 2 sections, separated by $2 \mathrm{~mm}$, were stained using haematoxylin and eosin for orientation purposes and for structural analysis. The right cerebral hemisphere was immersed in $20 \%$ sucrose in $\mathrm{PB}(0.1 \mathrm{M}$; pH 7.4), and then serial coronal sections $(40 \mu \mathrm{m})$ were cut on a freezing microtome; every 5 th section was stained with $0.01 \%$ thionin in acetate buffer ( $\mathrm{pH} 4.5)$. Intervening sections were collected and stored at $-20^{\circ} \mathrm{C}$ in cryoprotectant $(15 \%$ sucrose in $0.01 \mathrm{M} \mathrm{PB}$ containing ethylene glycol; $\mathrm{pH}$ 7.2) until required for free-floating, double-labelling immunofluorescence.

\section{Single-Labelling Immunohistochemistry}

All primary and secondary antibodies, dilutions and suppliers are summarised in table 1 . Immunohistochemistry was performed on paraffin-embedded sections to detect Ki67 (cells proliferating in late G1, S, G2, and M phases of cell cycle), von Willebrand factor (vWF; endothelial cells), polysialylated neuronal cell adhesion molecule (PSA-NCAM; immature neurons), glial fibrillary acidic protein (GFAP; astrocytes), and ionised calcium-binding adaptor molecule-1 (Iba-1; microglia) using techniques previously described [8]. When the primary antibody was replaced with PBS $(\mathrm{pH} 7.4)$ as a negative control, immunoreactivity failed to occur. For each antibody, all sections were stained simultaneously to ensure uniform conditions for subsequent analysis.

\section{Double-Labelling Immunofluorescence}

All primary and secondary antibodies, dilutions and suppliers are summarised in table 2 . Free-floating sections $(n=3)$ from the cerebral hemisphere (level of the frontal cortex) of control $(n=6)$ and GR $(n=7)$ foetuses were either immunostained sequentially 
Table 1. Single-labelling immunohistochemistry: primary and secondary antibodies

\begin{tabular}{llll}
\hline Primary antibody and dilution & Localisation & Supplier & Secondary antibody and dilution \\
\hline Rabbit anti-GFAP; 1:500 & Astrocytes & ZO334; DAKO, Calif., USA & Biotinylated anti-rabbit IgG; 1:200 \\
\hline Rabbit anti-Iba-1 ${ }^{1}$; 1:1,500 & Microglia & $\begin{array}{l}\text { 019-19741; Wako Pure Chemical } \\
\text { Industries, Osaka, Japan. }\end{array}$ & Biotinylated anti-rabbit IgG; 1:200 \\
\hline Mouse anti-human Ki67 $;$ 1:150 & Proliferating cells in late & M7240; DAKO, Calif., USA & Biotinylated anti-mouse IgG; 1:200 \\
\hline Rat anti-mouse PSA-NCAM; 1:250 & Immature neurons & 5A5; Hybridoma Bank, USA & Biotinylated anti-mouse IgM; 1:200 \\
\hline Rabbit anti-vWF ${ }^{2}$;:800 & Endothelial cells & Ab6994; Abcam, Mass., USA & Biotinylated anti-rabbit IgG; 1:200 \\
\hline
\end{tabular}

${ }^{1}$ Pre-treatment using citrate buffer ( $\mathrm{pH}$ 6) and microwaving. ${ }^{2}$ Pre-treatment using $0.02 \%$ proteinase $\mathrm{K}$ at $37^{\circ} \mathrm{C}$.

Table 2. Double-labelling immunofluorescence: primary and secondary antibodies

\begin{tabular}{llll}
\hline Primary antibody and dilution & Localisation & Supplier & Secondary antibody and dilution \\
\hline Rabbit anti-GFAP; 1:1,000 & Astrocytes & ZO334; DAKO, Calif., USA & Alexa Fluor 594 goat anti-rabbit; 1:500 \\
\hline Mouse anti-human Ki67; 1:1,000 & $\begin{array}{l}\text { Proliferating cells in late } \\
\text { G1, S, G2, and M phases }\end{array}$ & M7240; DAKO, Calif., USA & Alexa Fluor 488 donkey anti-mouse; 1:500 \\
\hline Mouse anti-nestin ; 1:500 & Immature neurons & MAB353; Millipore, Mass., USA & Alexa Fluor 594 donkey anti-mouse; 1:500 \\
\hline Rabbit anti-Olig2; 1:500 & Oligodendrocytes & AB9610; Millipore, Mass., USA & Alexa Fluor 594 goat anti-rabbit; 1:500 \\
\hline Rat anti-mouse PSA-NCAM ; 1:1,000 & Immature neurons & 5A5; Hybridoma Bank, USA & $\begin{array}{l}\text { Rhodamine (TRITC)-conjugated } \\
\text { AffiniPure } \\
\text { goat anti-mouse IgM; 1:500 }\end{array}$ \\
\hline
\end{tabular}

${ }^{1}$ Staining performed sequentially with Ki67.

(Ki67/PSA-NCAM and Ki67/nestin) or in parallel (Ki67/GFAP and Ki67/oligodendrocyte transcription factor 2, Olig2). For sequential immunofluorescence, sections were incubated with mouse anti-human Ki67 (1:1,000) and Alexa Fluor ${ }^{\circledR} 488$ donkey anti-mouse IgG (1:500; Molecular Probes, USA) followed by either rat anti-mouse PSA-NCAM (1:1,000) and Rhodamine (TRITC)conjugated AffiniPure goat anti-mouse IgM (1:500; Jackson ImmunoResearch, West Grove, Pa., USA) or mouse anti-nestin (1:500) and Alexa Fluor 594 donkey anti-mouse (1:500), as previously described [20]. Sections were blocked in $10 \%$ normal mouse serum in Tris-buffered saline for $1 \mathrm{~h}$ following the completion of Ki67 and prior to PSA-NCAM or nestin immunofluorescence to prevent cross-reactivity. For parallel immunofluorescence, sections were incubated with mouse anti-human Ki67 $(1: 1,000)$ together with rabbit anti-GFAP (1:1,000) or rabbit anti-Olig2 (1:500) followed by Alexa Fluor 488 donkey anti-mouse IgG (1:500) and Alexa Fluor 594 goat anti-rabbit IgG (1:500).

Analysis: Single-Labelling Immunohistochemistry

Volumetric and immunohistochemical analyses were performed using a calibrated Image Pro Plus software package (Version 4.1; Media Cybernetics, Rockville, Md., USA). Slides were coded in order to prevent experimenter bias. Means were calculated for each animal, and a mean of means for control and GR groups was determined.

Volume of the SVZ Occupied by Ki67-Immunoreactive Cells

The cross-sectional area of the dorsal SVZ (dSVZ) and ventral SVZ (vSVZ; fig. 1) occupied by Ki67-immunoreactive (IR) cells was measured in every 40 th section (12 sections per animal) with a digitizer interfaced to the software. As there are two clear regions of SVZ in the guinea pig at this gestational age we elected to measure them separately in the event that there was a differential response. The total volume of the dSVZ and of the vSVZ was then calculated according to the Cavalieri principle using the formula $\mathrm{V}=\Sigma \mathrm{APt}$, where $\mathrm{V}$ is the total volume, $\Sigma \mathrm{A}$ is the sum of the areas measured, $\mathrm{P}$ is the inverse of the sampling fraction and $\mathrm{t}$ is the section thickness [21].

Total Number of Ki67-IR Cells in the SVZ

Ki67-IR cells were counted in 3 random fields (objective: $100 \times$; field size: $2,480 \mu^{2}$ ) of the dSVZ and vSVZ in every 40 th section (12 sections per animal). Counts were expressed as cell density (counted as cells $/ \mathrm{mm}^{2}$ corrected to cells $/ \mathrm{mm}^{3}$ ), and the 
Fig. 1. Distribution of Ki67-IR cells within the SVZ in the foetal guinea pig brain. Ki67-positive cells were located within the dSVZ and vSVZ in both control (a) and GR (b) foetuses. Regions designated as dSVZ and vSVZ are outlined in black. Ki67-IR cells were located along the lateral (a, arrow) and medial (a, arrowhead) edges on the LV; there was a greater density of cells along the lateral edge. Ki67-IR cells were occasionally located within the ependymal layer (a, inset). Scale bar $(\mathbf{a}, \mathbf{b})=130 \mu \mathrm{m}$; inset $(\mathbf{a})=50 \mu \mathrm{m}$.
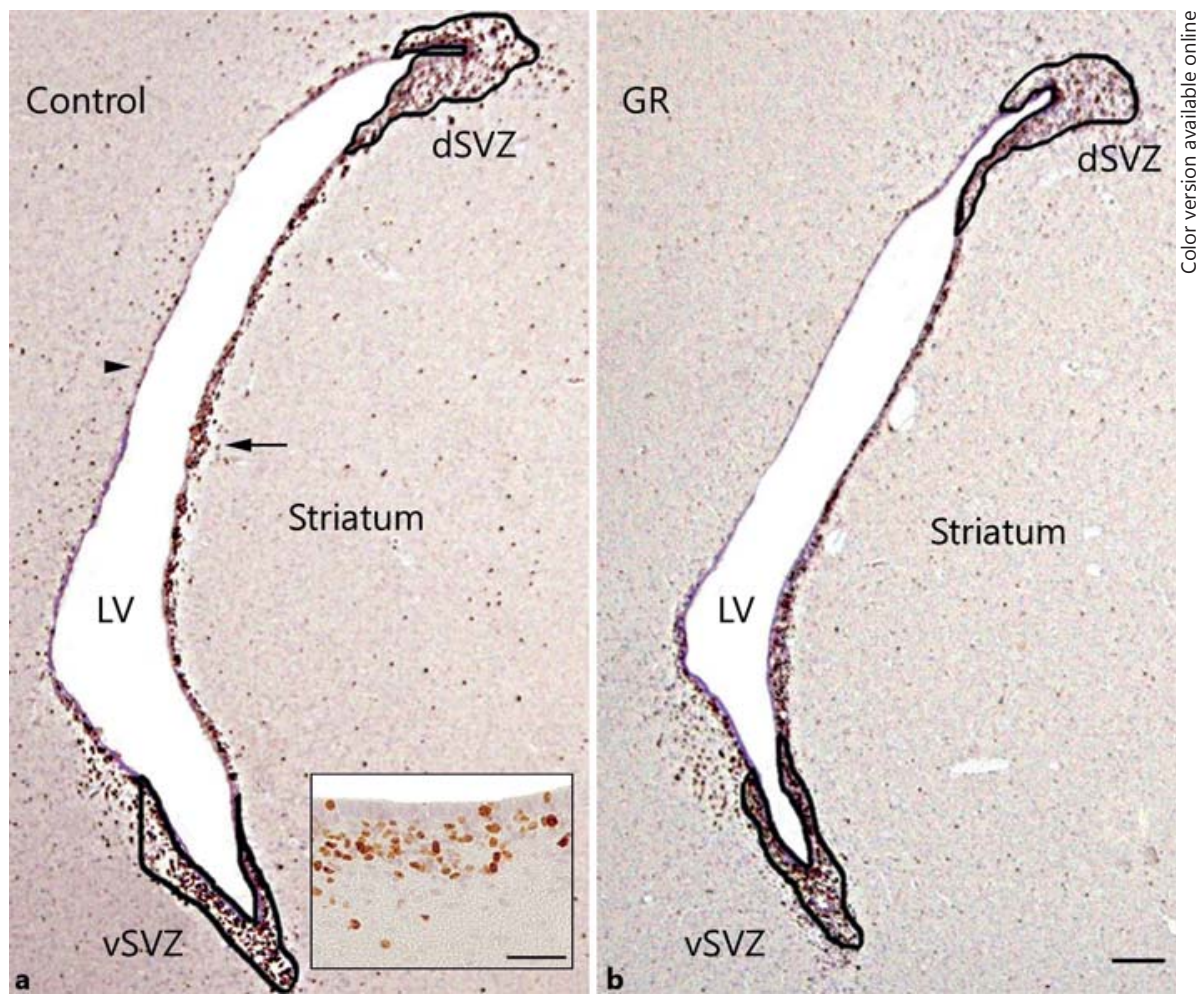

total number of Ki67-IR cells for each animal was determined by multiplying cell density $\left(\mathrm{mm}^{3}\right)$ by the volume of the $\mathrm{dSVZ}$ and vSVZ.

Assessment of Blood Vessels in the SVZ

The percentage of the dSVZ and vSVZ occupied by vWF-IR blood vessels was assessed in 2 rostral and 2 caudal sections (each separated by $80 \mu \mathrm{m} ; 4$ sections per animal) of the cerebral hemisphere using a point-counting technique [22].

Cell Density of Iba-1-IR Microglia in the SVZ

Iba-1-IR microglia were counted in the dSVZ and vSVZ in 2 rostral and 2 caudal sections (each separated by $160 \mu \mathrm{m} ; 4$ sections per animal) of the cerebral hemisphere. The cross-sectional area of the dSVZ and vSVZ was measured in each section using a digitizer interfaced to software, and data were expressed as cells $/ \mathrm{mm}^{2}$.

Qualitative Analysis of PSA-NCAM- and GFAP-IR in the SVZ

2 rostral and 2 caudal sections (each separated by $80 \mu \mathrm{m} ; 4$ sections per animal) immunostained for PSA-NCAM and GFAP were digitally scanned using the MIRAX Digital Slide scanner (Zeiss, Australian Phenomics Network). Qualitative assessment of the staining was then performed using MIRAX Viewer software.

Analysis: Double-Labelling Immunofluorescence

Sections of the frontal cortex were examined using confocal microscopy (Zeiss Meta confocal laser scanning system or Nikon C1 inverted confocal microscope). Fluorophores were vi- sualised using an excitation filter of $488 \mathrm{~nm}$ and an emission filter of 522/535 nm for Alexa 488, and an excitation filter of 568 $\mathrm{nm}$ and an emission filter of $605 / 632 \mathrm{~nm}$ for Rhodamine (TRITC). Optical images were acquired using a $40 \times$ or $63 \times$ oil immersion lens.

Semi-Quantitative Assessment of the Phenotype of

Proliferating Cells

In the sections of the frontal cortex from each of the control and IUGR foetuses, the dSVZ and vSVZ were assessed semi-quantitatively for co-localisation of Ki67 with nestin, GFAP and Olig2 ( $40 \times ; 2$ fields of view per section for both the dSVZ and vSVZ).

\section{Statistical Analysis}

Body and brain weight data were expressed as the mean of means \pm SD; all other data were expressed as the mean of means \pm SEM. All analyses were performed using a Student's t test using SigmaPlot 12 Statistical Software (Systat Software Inc., San Jose, Calif., USA) unless a test for normality failed, in which case a Mann-Whitney U test was performed. Data were considered significant when $\mathrm{p}<0.05$. Linear regression analysis (including both groups of animals) was carried out to determine whether there was a correlation between brain weight (as an indication of GR) and the following: (1) the total number of Ki67-IR cells in the dSVZ and vSVZ; (2) the percentage of the dSVZ and vSVZ occupied by blood vessels, and (3) microglial density in the dSVZ and vSVZ. In addition, regression analysis was performed to test for a correlation between the total number of Ki67-IR cells and the percentage of dSVZ and vSVZ occupied by blood vessels. 
Table 3. Body and brain weights of control and IUGR guinea pigs at $60 \mathrm{DG}$

\begin{tabular}{lcc}
\hline & Control $(\mathrm{n}=6)$ & IUGR $(\mathrm{n}=7)$ \\
\hline Body weight, g & $103.03 \pm 12.75$ & $50.23 \pm 12.27^{*}$ \\
Brain weight, g & $2.71 \pm 0.18$ & $2.15 \pm 0.19^{*}$ \\
Crown-rump length, cm & $13.78 \pm 0.47$ & $10.33 \pm 1.06^{*}$ \\
Brain:body ratio & $0.03 \pm 0.003$ & $0.04 \pm 0.009^{*}$ \\
\hline
\end{tabular}

Values are expressed as means $\pm \mathrm{SD} .{ }^{*} \mathrm{p}<0.005$, compared to age-matched controls (Mann-Whitney $U$ test).

\section{Results}

\section{Body and Brain Weights}

At 60 DG, there was a decrease $(p<0.005)$ in body weight, brain weight and crown-rump length in GR foetuses $(n=7)$ compared to controls $(n=6$; table 3$)$. The ratio of brain-to-body weight was increased $(\mathrm{p}<0.005)$ in GR foetuses compared to controls (table 3 ), reflecting the relative sparing of the brain.

\section{Cell Proliferation in the SVZ}

In control and GR guinea pigs at $60 \mathrm{DG}$, dense accumulations of Ki67-IR cells were present at both the dorsal and ventral aspects of the LV (fig. 1a, b). Ki67-IR cells, extending several cells thick from the ependymal layer, were also present along the lateral edge of the LV (apposed to the striatum; fig. 1a, arrow); there were fewer Ki67-IR cells along the medial edge of the LV (fig. 1a, arrowhead). In both groups, Ki67-IR cells were occasionally located within the ependymal cell layer at the dorsal and ventral aspects of the LV, either as individual cells or small clusters of cells (fig. 1a, inset).

At 60 DG, there was no difference ( $p>0.05$ ) between control and GR foetuses in the mean volumes of the dSVZ or vSVZ occupied by Ki67-IR cells (fig. 2a) or in the total number of Ki67-IR cells in these regions (fig. 2b). Regression analysis across all animals revealed a negative correlation between brain weight and the number of Ki67-IR cells in the vSVZ $\left(p<0.05 ; \mathrm{r}^{2}=0.33\right.$; fig. $\left.2 \mathrm{~d}\right)$; this is illustrated by comparing the vSVZ in controls (fig. 2e), GR foetuses (fig. 2f) and severely GR foetuses (fig. 2g). There was no correlation in the dSVZ $\left(\mathrm{p}=0.15 ; \mathrm{r}^{2}=0.18\right.$; fig. 2c).

In both the control (fig. 3a-c) and IUGR foetuses (data not shown), double-labelling immunofluorescence revealed that Ki67-IR in the dSVZ (and vSVZ; data not shown) co-localised with PSA-NCAM-IR (approximate-

Cell Proliferation and Angiogenesis in the

Foetal SVZ in Growth Restriction ly 95\%; fig. 3a) and nestin-IR (fig. 3b) but did so less frequently (approximately 5\%) with Olig2-IR (fig. 3c). In both the control (fig. 3d) and IUGR foetuses (data not shown), there was a meshwork of GFAP-IR fibre staining in the region of the dSVZ occupied by Ki67-IR cells; however, GFAP-IR cell bodies were not co-localised with Ki67-IR cells.

\section{Vascular and Cellular Microenvironment in the SVZ}

Microvasculature

There was no difference $(\mathrm{p}>0.05)$ in the mean percentage of the dSVZ and vSVZ occupied by blood vessels in the control and GR foetuses (fig. 4a). However, regression analysis revealed a negative correlation between brain weight and the percentage of the vSVZ occupied by blood vessels $\left(p=0.05 ; r^{2}=0.29\right)$; this correlation was not significant for the dSVZ $\left(p>0.05 ; r^{2}=0.11\right)$.

There was a positive correlation between the total number of Ki67-IR cells and the percentage of the dSVZ ( $p<0.05 ; \mathrm{r}^{2}=0.33$; fig. $\left.4 \mathrm{~b}\right)$ and vSVZ ( $<0.0001$; $\mathrm{r}^{2}=0.81$; fig. $4 \mathrm{c}$ ) occupied by blood vessels; this is illustrated by comparing the vSVZ in controls (fig. $4 \mathrm{~d}$ ), moderately GR foetuses (fig. 4e) and severely GR foetuses (fig. 4f).

\section{Microglia}

There was no difference $(\mathrm{p}>0.05)$ in the density of Iba-1-IR microglia in the dSVZ or vSVZ in the control and GR foetuses (fig. 5a), as illustrated by comparing controls (fig. 5b) and GR foetuses (fig. 5c). Regression analysis revealed no correlation ( $p>0.05$ ) between brain weight and the density of Iba-1-IR microglia in the dSVZ or vSVZ (data not shown). The morphology of microglia (e.g. size of the cell body, process length or thickness) was also not different between groups.

\section{Immature Neurons}

PSA-NCAM-IR was present within the dSVZ and vSVZ in the control and GR foetuses (fig. 6a-d). In the dSVZ, immunoreactivity was localised to the cytoplasm of cell bodies (fig. 6a, inset) and to fibre bundles located adjacent and lateral to the SVZ (fig. $6 \mathrm{a}-\mathrm{c}$ ); the density of positive cells and the intensity of staining was more pronounced in the rostral (fig. 6a) than the caudal sections (fig. 6b). In the vSVZ, PSA-NCAM-IR was present at a similar intensity in the rostral (fig. 6d) and caudal sections (data not shown). There appeared to be no difference in immunoreactivity between the controls (fig. 6b) and GR foetuses (fig. 6c; dSVZ at the caudal level). 


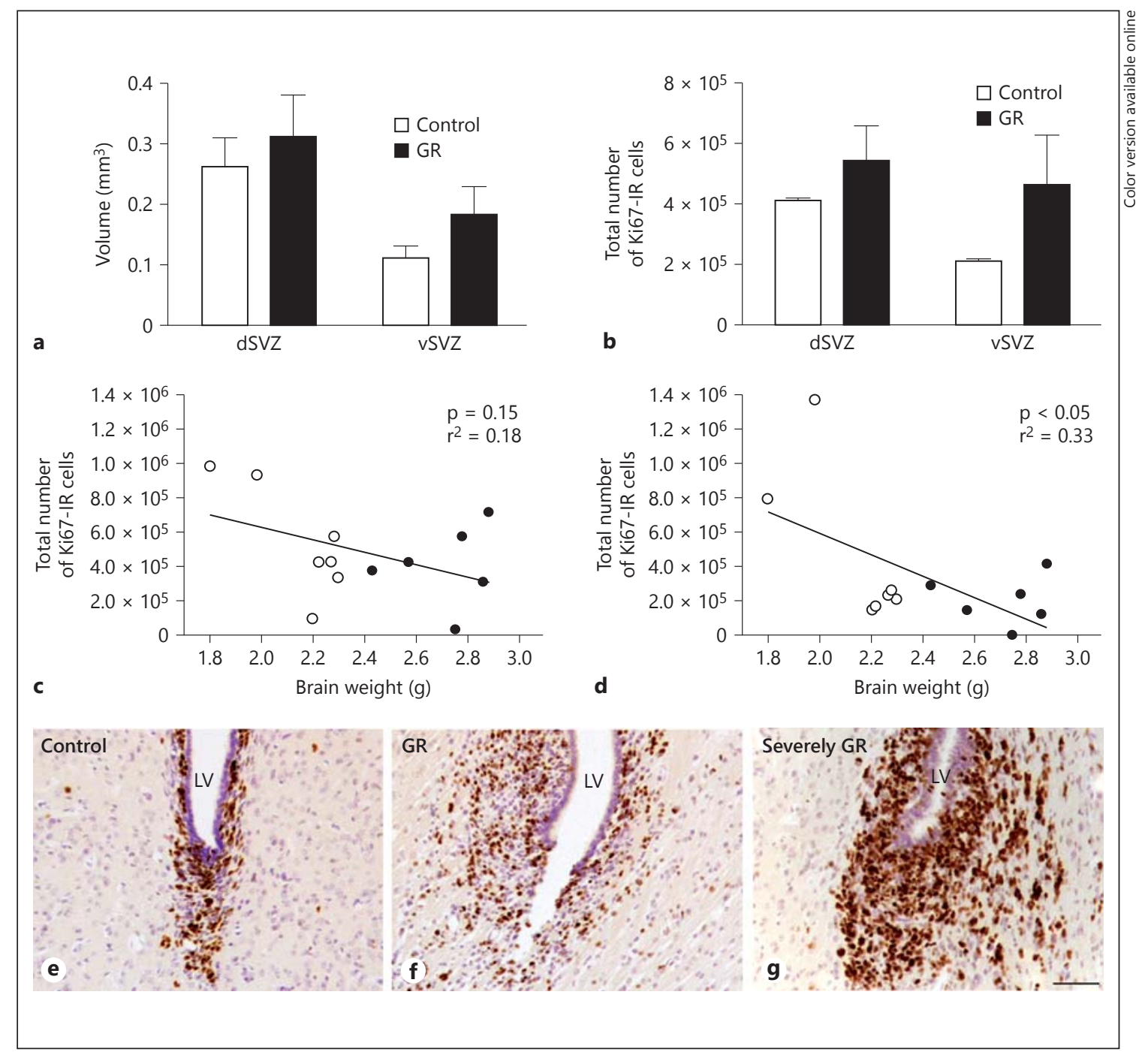

Fig. 2. Analysis of cell proliferation in the dSVZ and vSVZ in control and GR foetuses. There was no difference in the mean volumes (a) or the total number of Ki67-IR cells (b) in the dSVZ and vSVZ in control and GR foetuses. Regression analysis across all animals revealed a negative correlation $(\mathrm{p}<0.05)$ between brain weight and the number of Ki67-IR cells in the vSVZ (d), but there was no difference $(\mathrm{p}>0.05)$ in the dSVZ $(\mathbf{c})$. The negative correlation between brain weight and the number of Ki67-IR cells in the vSVZ is illustrated by comparing images from control (e), GR (f) and severely GR (g) foetuses. Scale bar $(\mathbf{e}-\mathbf{g})=60 \mu \mathrm{m}$.

\section{Astrocytes}

In the dSVZ, GFAP-IR was present predominantly in the caudal sections (fig. 6f) but was occasionally evident in the rostral sections, associated with a high density of Ki67-IR cells (fig. 6e); there was an absence of GFAP-IR in the vSVZ at both levels (fig. 6h; rostral level). There appeared to be no difference in the density of GFAP-IR in the dSVZ between the control (fig. 6f) and GR (fig. 6g) foetuses and no correlation between the amount of GFAPIR and severity of GR. There was no evidence of reactive morphological changes in the astrocytes.

\section{Discussion}

Here we assess for the first time the impact of IUGR, induced by CPI throughout the second half of gestation, on neural precursor cells and the microenvironment in the SVZ of the foetal guinea pig. Key findings were, firstly, that with decreasing brain weight both the number of proliferating cells in the vSVZ and the percentage of $\mathrm{vSVZ}$ occupied by blood vessels increased. This effect was not significant in the dSVZ, suggesting that there might be a differential response between the regions, although we 

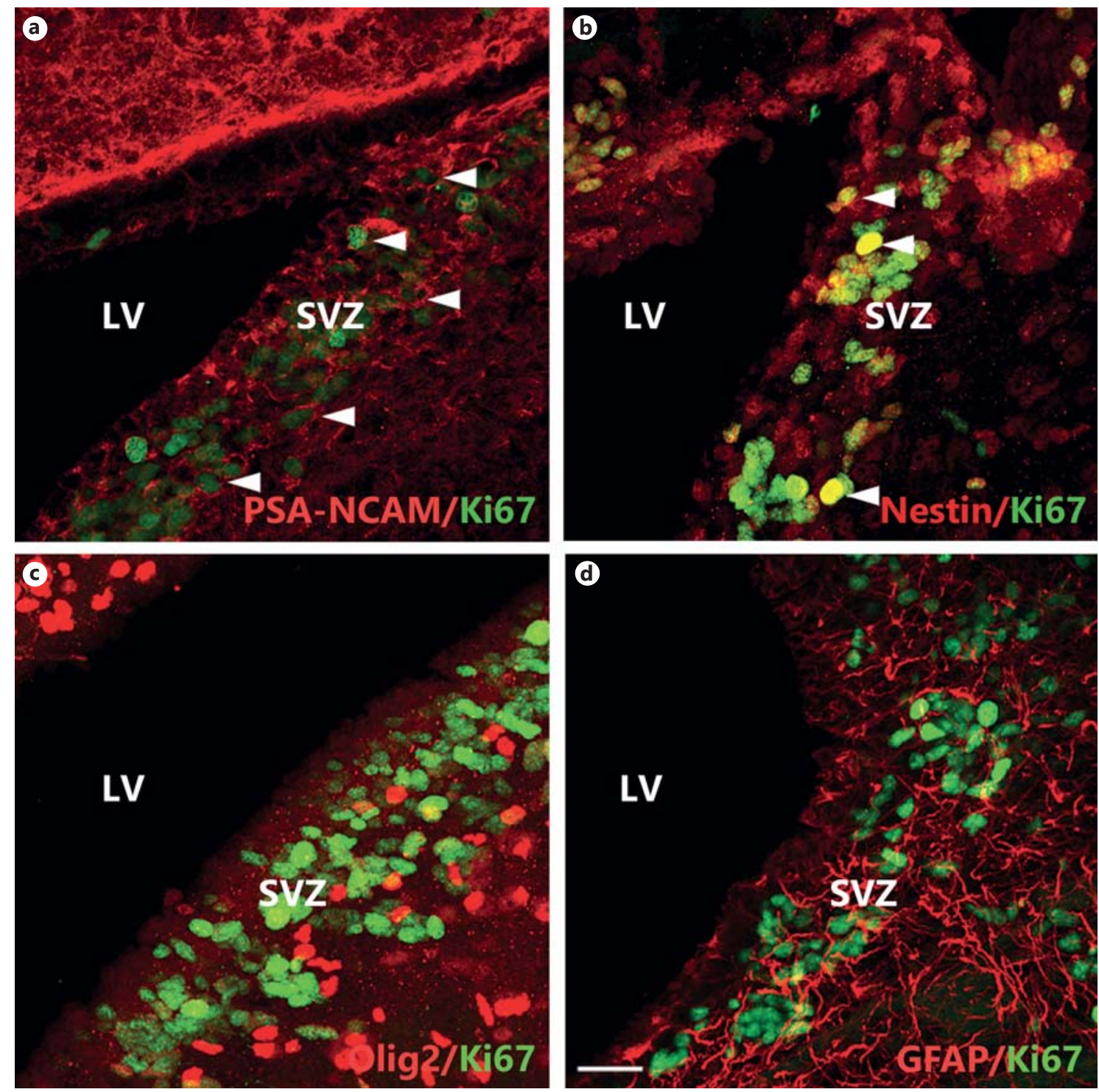

Fig. 3. Representative image from a control guinea pig foetus showing phenotypic analysis of Ki67-IR proliferating cells in the dSVZ. Ki67-IR proliferating cells co-localised with cytoplasmic PSA-NCAM-IR (a, arrowheads) and nestin-IR cells (b, arrow- heads). There was little co-localisation between Ki67-IR and Olig2-IR cells (c) and none with Ki67-IR and GFAP-IR cell bodies (d). However, GFAP-IR astrocytic processes extended throughout the SVZ. Scale bar $(\mathbf{a}-\mathbf{d})=20 \mu \mathrm{m}$. currently have no explanation for this. Co-localisation studies revealed that Ki67-IR cells were predominantly immature neurons - a small proportion of these were identified as oligodendroglia. Secondly, as the percentage of dSVZ and vSVZ occupied by blood vessels increased there was a concomitant increase in the number of Ki67 cells. This finding suggests that CPI alters the components of the neurogenic/neurovascular microenvironment and that the microvasculature and cell proliferation in the SVZ are closely aligned.

Cell Proliferation and Angiogenesis in the Foetal SVZ in Growth Restriction
Increased Cell Proliferation in the SVZ and

Correlation with Decreasing Brain Weight

Several studies have highlighted the capacity of neural progenitor cells within the neonatal SVZ to respond to injury as a result of hypoxia or hypoxia-ischaemia and have reported both an increase [10-14] and a decrease [15-17] in proliferation. Relatively little, however, is known concerning the foetus. It is to be expected that there will be some variability in outcome following CPI, reflecting the highly specific intrauterine environment

Dev Neurosci 2015;37:453-463 


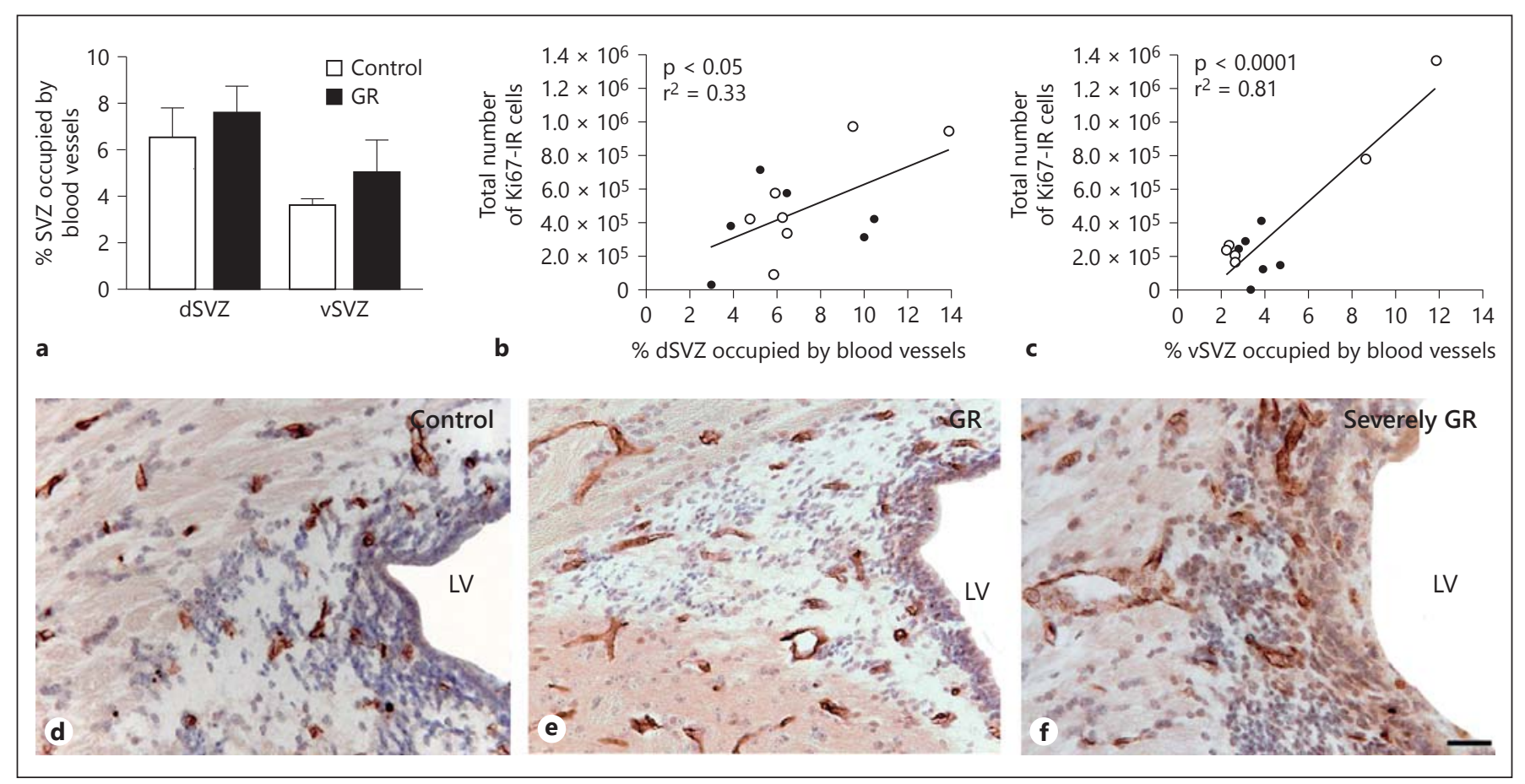

Fig. 4. Analysis of the microvasculature and cell proliferation in the dSVZ and vSVZ in control and GR foetuses. a There was no significant difference in the mean percentage of dSVZ or vSVZ occupied by vWF-IR blood vessels in control and GR foetuses. There was a positive correlation between the total number of Ki67-IR cells and the percentage of the dSVZ (b) and vSVZ (c) occupied by vWF-IR blood vessels. This is illustrated by comparing images of vWF immunoreactivity in the dSVZ from control (d), GR (e) and severely GR (f) foetuses. Scale bar (d-f) $=40 \mu \mathrm{m}$.

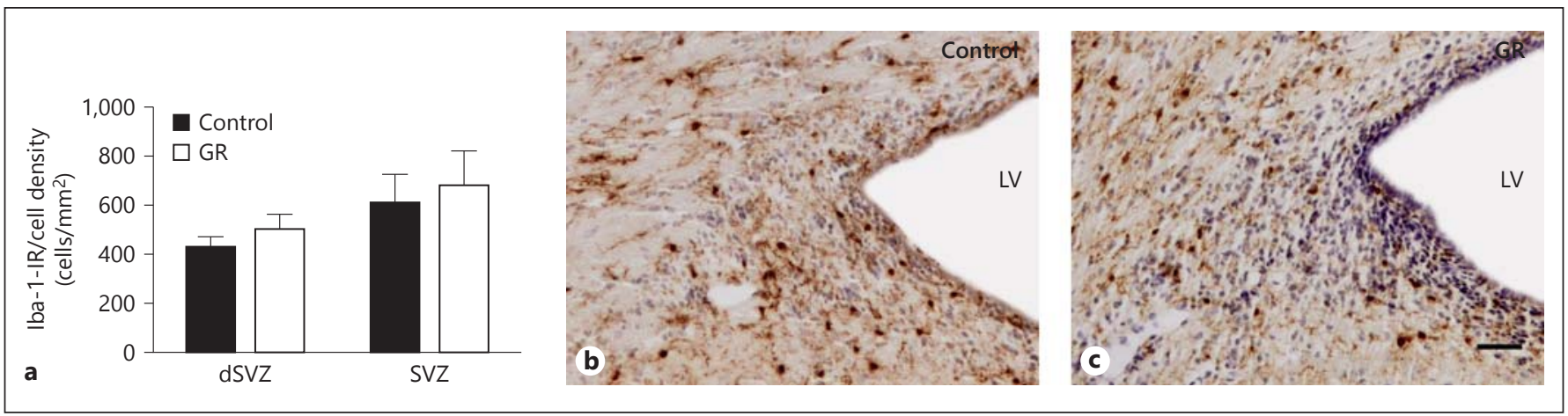

Fig. 5. Analysis of microglia within the dSVZ and vSVZ of control and GR foetuses. a There was no significant difference in the mean density (cells $/ \mathrm{mm}^{2}$ ) of Iba-1-IR microglia in the dSVZ or vSVZ

for each foetus within the paradigm of compromise. Here we show that CPI resulting in IUGR might be a trigger for increased neural cell precursor proliferation in the SVZ, given the correlation between proliferation in the vSVZ and the severity of GR, as evidenced by decreasing brain weight. We acknowledge that by combining moderately and severely GR foetuses in one group we between control and GR foetuses. This is illustrated by comparing images of Iba-1-IR cells in the dSVZ from control (b) and GR (c) foetuses. Scale bar (b, c) $=40 \mu \mathrm{m}$.

might be omitting to distinguish between the effects of moderate versus severe GR; such a difference has been reported for myelination in a rodent model of GR [23]. Animal numbers in the present study precluded such an analysis, but we add the caveat that the findings we report here appear to be most pronounced in the severely GR foetuses. 


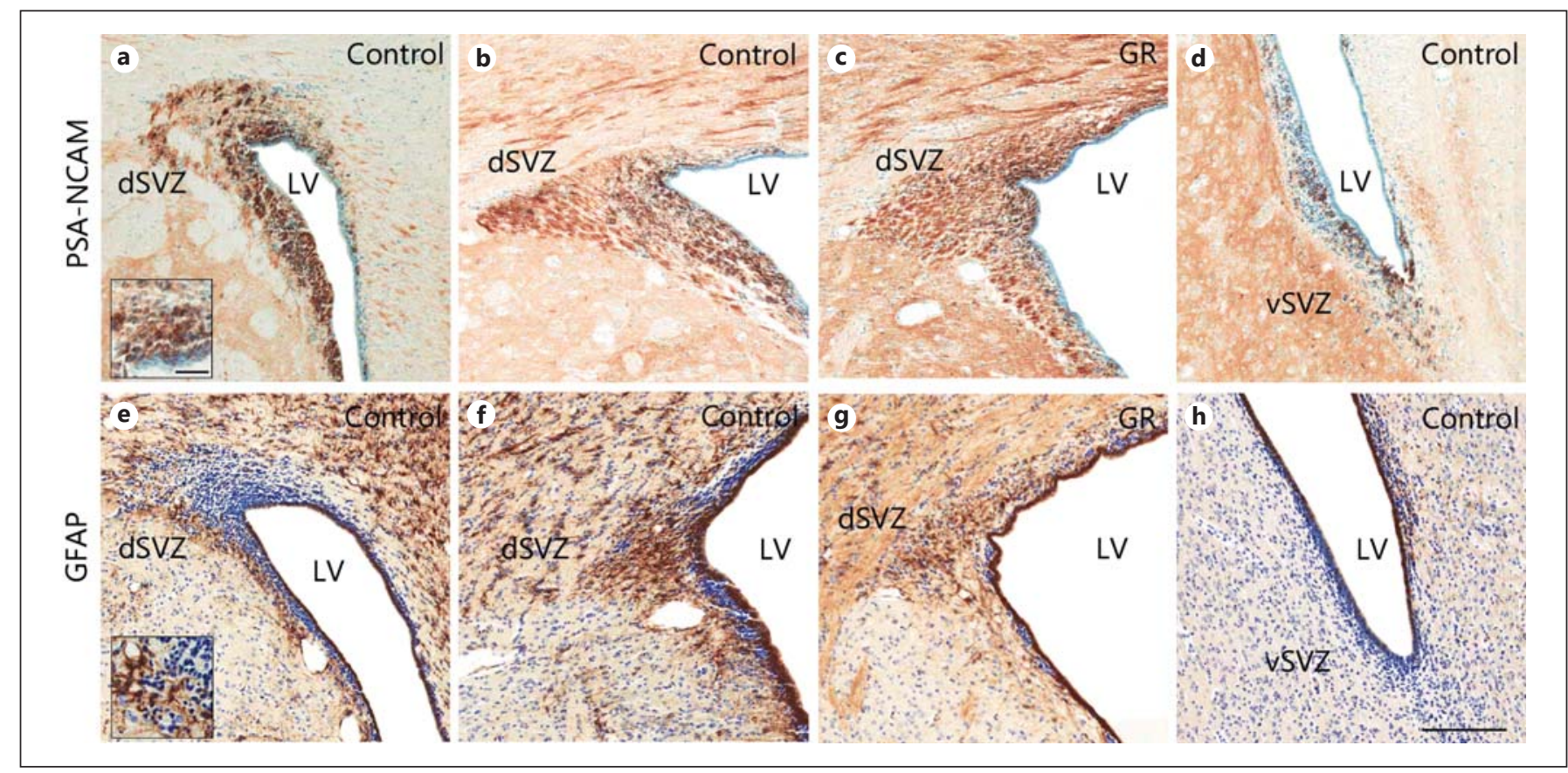

Fig. 6. PSA-NCAM (a-d) and GFAP immunoreactivity (e-h) within the dSVZ and vSVZ in control and GR foetuses. Immunoreactivity for PSA-NCAM was present in the dSVZ of control (a, b) and GR foetuses (c) in sections from the rostral (a) and caudal levels $(\mathbf{b}, \mathbf{c})$ of the brain. There appeared to be no difference in the density or intensity of PSA-NCAM immunoreactivity in control (b) vs. GR foetuses (c). d In the vSVZ, PSA-NCAM-IR was present at a similar intensity at both the caudal and rostral levels of the brain (rostral level shown). Immunoreactivity for GFAP was pres-

In relation to the sequence of events in the SVZ, there might be an initial reduction in cell proliferation and an increase in cell death in the period immediately after the induction of CPI, followed by cell proliferation as a compensatory mechanism. Such a response has been shown following acute hypoxia in perinatal rats [15]. Assessment of cell death at foetal ages closer to the time of insult in the guinea pig would be required to validate this theory. In addition to hypoxaemia [24], CPI in the guinea pig results in hypoglycaemia [19] and altered levels of cortisol in GR foetuses [19], parameters which, in other species, have been shown to influence cell proliferation $[25,26]$. Thus, it is possible that an altered endocrine status might also play a role in influencing cell proliferation in the GR foetus.

\section{Angiogenesis in the SVZ Is Correlated with Increasing} Cell Proliferation and Decreasing Brain Weight

It is now acknowledged that the cerebral microenvironment in which a neural precursor cell finds itself ent in the dSVZ of control (e, f, inset in $\mathbf{e})$ and GR foetuses $(\mathbf{g})$ in sections from the rostral (e, inset in $\mathbf{e}$ ) and caudal levels $(\mathbf{f}, \mathbf{g})$ of the brain. There appeared to be no difference in the density of GFAP immunoreactivity in control (f) vs. GR foetuses (g). GFAP immunoreactivity was absent in the vSVZ in both control (h) and GR (data not shown) foetuses at both caudal (data not shown) and rostral levels (h) of the brain compared to the dSVZ (e). Scale bar $(\mathbf{a}-\mathbf{h})=170 \mu \mathrm{m}$; inset $(\mathbf{a}, \mathbf{e})=60 \mu \mathrm{m}$.

needs to be conducive to proliferation. Astrocytes, activated microglia, progenitor cells and, importantly, the microvasculature, constitute a so-called neurogenic or vascular niche [27]. The importance of a neurogenic niche has predominantly been described for adult neurogenesis. However, there is now evidence that intermediate progenitors in the embryonic SVZ require a neurogenic niche for proliferation [28]. During embryonic cortical development, intermediate progenitor cells in the neurogenic niche are spatially and temporally associated with blood vessels and divide near blood vessel branch points [28]. Furthermore, the position of progenitor cells during proliferation, migration and differentiation is correlated with the development of endothelial cells in the SVZ [28]. Taken together, this suggests that angiogenesis and neurogenesis are developmentally coordinated processes and provides evidence of a neurogenic niche in the foetal SVZ. In the present study, we have demonstrated a significant correlation between angiogenesis and increased neural precursor cell prolifera- 
tion, supporting the concept of a foetal neurogenic niche. Furthermore, we have shown that angiogenesis in the vSVZ increases as brain weight decreases, suggesting that the more severe the GR the greater the proliferation of blood vessels. Hypoxic-ischaemic insults are known to cause dilatation of blood vessels and promote angiogenesis in other regions of the perinatal brain $[29,30]$, and it is likely that hypoxaemia plays a role here.

Other cellular components of the niche, including microglia and astrocytes, were unaffected in IUGR and were not correlated with the degree of foetal GR, suggesting that, in our model, the microvasculature is the most critical component of the foetal neurogenic niche at this stage of development. Neurotrophins such as brain-derived neurotrophic factor and growth factors such as vascular endothelial growth factor and fibroblast growth factor 2 are released from endothelial cells $[31,32]$ and influence neuronal survival, proliferation and differentiation [33]. Thus, an increased release of these growth factors, due to an increase in angiogenesis, may contribute to the foetal neurogenic niche and mediate the proliferative effect; this warrants further investigation.

\section{Long-Term Consequences of Increased Neurogenesis \\ in the SVZ in Severely GR Foetuses}

Despite the increase in cell proliferation within the SVZ in severely GR foetuses, we know from our previous investigations that the volume of the neocortex is actually reduced in IUGR guinea pigs 1 week postnatally [8], with reductions in the volume of the basal ganglia and septal area at 8 weeks [34]. This suggests that neural precursor cells fail to differentiate and survive postnatally and/or that the growth of their processes is reduced. In other regions of the brain in this model we have demonstrated reduced neuronal numbers [7] and reduced dendritic morphology [35] postnatally.

\section{Limitations of the Study}

Cell proliferation and angiogenesis were only assessed at one foetal age (60 DG) and, thus, we are unable to comment on the immediate effects of CPI or on the trajectory of the response. Examining other time points between 30 DG (the time of the insult) and 60 DG would address this limitation. Neither can we comment specifically on the fate of the proliferating cells postnatally, as indicated above; fate-mapping studies could provide this data but were outside the scope of this study.

\section{Conclusion}

We have found that there is an increase in neural precursor cell proliferation and density of blood vessels in the $\mathrm{vSVZ}$ of compromised foetuses and that this is negatively correlated with the extent of GR. Furthermore, blood vessel density increases concomitantly with cell proliferation throughout the SVZ. We suggest that these represent intrinsic mechanisms designed to counter the effects of CPI. Although our previous studies indicate that enhanced neurogenesis is unlikely to be sustained in the long term following CPI, we have demonstrated that there is a window of opportunity in which to deliver appropriate trophic support in order to improve neurological outcome.

\section{Acknowledgements}

The authors wish to thank Mr. Todd Briscoe for his surgical assistance, Ms. Nadia Hale for her confocal microscopy expertise and Ms. Aminath Azhan for technical assistance.

Funding was provided by the National Health and Medical Research Council of Australia (Project Grant 454536 awarded to M.T., S.R. and A.T.) and the Victorian Government's Operational Infrastructure Support Program (Government of Victoria).

\section{References}

1 Geva R, Eshel R, Leitner Y, Valevski AF, Harel S: Neuropsychological outcome of children with intrauterine growth restriction: a 9-year prospective study. Pediatrics 2006;118:91100.

- 2 Esteban FJ, Padilla N, Sanz-Cortes M, de Miras JR, Bargallo N, Villoslada P, Gratacos E: Fractal-dimension analysis detects cerebral changes in preterm infants with and without intrauterine growth restriction. Neuroimage 2010;53:1225-1232.

3 Smedler AC, Faxelius G, Bremme K, Lagerstrom M: Psychological development in chil- dren born with very low birth weight after severe intrauterine growth retardation: a $10-$ year follow-up study. Acta Paediatr 1992;81: 197-203.

4 Miller J, Turan S, Baschat AA: Fetal growth restriction. Semin Perinatol 2008;32:274-280.

5 Salam RA, Das JK, Bhutta ZA: Impact of intrauterine growth restriction on long-term health. Curr Opin Clin Nutr Metab Care 2014;17:249-254.

6 Peebles DM: Fetal consequences of chronic substrate deprivation. Semin Fetal Neonatal Med 2004;9:379-386.
7 Mallard C, Loeliger M, Copolov D, Rees S: Reduced number of neurons in the hippocampus and the cerebellum in the postnatal guinea-pig following intrauterine growth restriction. Neuroscience 2000;100:327333.

8 Tolcos M, Bateman E, O’Dowd R, Markwick $R$, Vrijsen K, Rehn A, Rees S: Intrauterine growth restriction affects the maturation of myelin. Exp Neurol 2011;232:53-65.

-9 Kriegstein A, Alvarez-Buylla A: The glial nature of embryonic and adult neural stem cells. Annu Rev Neurosci 2009;32:149-184. 
10 Fagel DM, Ganat Y, Silbereis J, Ebbitt T, Stewart W, Zhang $\mathrm{H}$, Ment LR, Vaccarino FM: Cortical neurogenesis enhanced by chronic perinatal hypoxia. Exp Neurol 2006;199:7791.

-11 Felling RJ, Snyder MJ, Romanko MJ, Rothstein RP, Ziegler AN, Yang Z, Givogri MI, Bongarzone ER, Levison SW: Neural stem/ progenitor cells participate in the regenerative response to perinatal hypoxia/ischemia. J Neurosci 2006;26:4359-4369.

12 Yang Z, Covey MV, Bitel CL, Ni L, Jonakait GM, Levison SW: Sustained neocortical neurogenesis after neonatal hypoxic/ischemic injury. Ann Neurol 2007;61:199-208.

$\checkmark 13$ Yang Z, Levison SW: Hypoxia/ischemia expands the regenerative capacity of progenitors in the perinatal subventricular zone. Neuroscience 2006;139:555-564.

14 Yang Z, Levison SW: Perinatal hypoxic/ischemic brain injury induces persistent production of striatal neurons from subventricular zone progenitors. Dev Neurosci 2007;29:331340.

15 Levison SW, Rothstein RP, Romanko MJ, Snyder MJ, Meyers RL, Vannucci SJ: Hypox$\mathrm{ia} /$ ischemia depletes the rat perinatal subventricular zone of oligodendrocyte progenitors and neural stem cells. Dev Neurosci 2001;23: 234-247.

-16 Romanko MJ, Zhu C, Bahr BA, Blomgren K, Levison SW: Death effector activation in the subventricular zone subsequent to perinatal hypoxia/ischemia. J Neurochem 2007;103: 1121-1131.

-17 Spadafora R, Gonzalez FF, Derugin N, Wendland M, Ferriero D, McQuillen P: Altered fate of subventricular zone progenitor cells and reduced neurogenesis following neonatal stroke. Dev Neurosci 2010;32:101113.
18 Tolcos M, Rees S: Chronic placental insufficiency in the fetal guinea pig affects neurochemical and neuroglial development but not neuronal numbers in the brainstem: a new method for combined stereology and immunohistochemistry. J Comp Neurol 1997;379: 99-112.

19 Jones CT, Lafeber HN, Roebuck MM: Studies on the growth of the fetal guinea pig. Changes in plasma hormone concentration during normal and abnormal growth. J Dev Physiol 1984;6:461-472.

20 Munro K, Rees S, O’Dowd R, Tolcos M: Developmental profile of erythropoietin and its receptor in guinea-pig retina. Cell Tissue Res 2009;336:21-29.

21 Gundersen HJ, Jensen EB: The efficiency of systematic sampling in stereology and its prediction. J Microsc 1987;147:229-263.

22 Tolcos M, Harding R, Loeliger M, Breen S, Cock M, Duncan J, Rees S: The fetal brainstem is relatively spared from injury following intrauterine hypoxemia. Brain Res Dev Brain Res 2003; 143:73-81.

23 Olivier P, Baud O, Bouslama M, Evrard P, Gressens P, Verney C: Moderate growth restriction: deleterious and protective effects on white matter damage. Neurobiol Dis 2007;26: 253-263.

24 Jones CT, Parer JT: The effect of alterations in placental blood flow on the growth of and nutrient supply to the fetal guinea-pig. J Physiol 1983;343:525-537.

25 Coe CL, Kramer M, Czeh B, Gould E, Reeves AJ, Kirschbaum C, Fuchs E: Prenatal stress diminishes neurogenesis in the dentate gyrus of juvenile rhesus monkeys. Biol Psychiatry 2003;54:1025-1034.

26 Suh SW, Fan Y, Hong SM, Liu Z, Matsumori Y, Weinstein PR, Swanson RA, Liu J: Hypoglycemia induces transient neurogenesis and subsequent progenitor cell loss in the rat hippocampus. Diabetes 2005;54:500-509.
27 Lichtenwalner RJ, Parent JM: Adult neurogenesis and the ischemic forebrain. J Cereb Blood Flow Metab 2006;26:1-20.

-28 Javaherian A, Kriegstein A: A stem cell niche for intermediate progenitor cells of the embryonic cortex. Cereb Cortex 2009; 19(suppl 1):i70-i77.

29 Brand MM, Bignami A: The effects of chronic hypoxia on the neonatal and infantile brain. A neuropathological study of five premature infants with the respiratory distress syndrome treated by prolonged artificial ventilation. Brain 1969;92:233-254.

30 Mallard EC, Rees S, Stringer M, Cock ML, Harding R: Effects of chronic placental insufficiency on brain development in fetal sheep. Pediatr Res 1998;43:262-270.

-31 Guo S, Kim WJ, Lok J, Lee SR, Besancon E, Luo BH, Stins MF, Wang X, Dedhar S, Lo EH: Neuroprotection via matrix-trophic coupling between cerebral endothelial cells and neurons. Proc Natl Acad Sci U S A 2008;105:7582-7587.

32 Shen Q, Goderie SK, Jin L, Karanth N, Sun Y, Abramova N, Vincent P, Pumiglia K, Temple $S$ : Endothelial cells stimulate self-renewal and expand neurogenesis of neural stem cells. Science 2004;304:1338-1340.

33 Kim BK, Kim SE, Shim JH, Woo DH, Gil JE, Kim SK, Kim JH: Neurogenic effect of vascular endothelial growth factor during germ layer formation of human embryonic stem cells FEBS Lett 2006;580:5869-5874.

- 34 Rehn AE, Van Den Buuse M, Copolov D, Briscoe T, Lambert G, Rees S: An animal model of chronic placental insufficiency: relevance to neurodevelopmental disorders including schizophrenia. Neuroscience 2004; 129:381-391.

35 Dieni S, Rees S: Dendritic morphology is altered in hippocampal neurons following prenatal compromise. J Neurobiol 2003;55:41-52. 